\title{
Revisions of Extensive Acetabular Defects with Impaction Grafting and a Cement Cup
}

\author{
Nienke van Egmond MD, Daniel C. J. De Kam MD, \\ Jean W. M. Gardeniers MD, PhD, \\ B. Willem Schreurs MD, PhD
}

Received: 11 December 2009/ Accepted: 24 September 2010/Published online: 8 October 2010

(C) The Author(s) 2010. This article is published with open access at Springerlink.com

\begin{abstract}
Background Loosening of acetabular components often leads to bony defects. Management of extensive acetabular bone loss in hip revision arthroplasty can be a tremendous challenge.

Questions/purposes We asked whether a reconstruction with impacted bone grafts will provide a durable and painfree function in extensive acetabular defects. We specifically determined the (1) survival rates with the end point of revision for any reason, aseptic revision, and radiographic loosening; (2) visual analog scale (VAS) pain score, Harris hip score (HHS), and the Oxford Hip Questionnaire score (OHQS); (3) number of repeat revisions; (4) complications; and (5) radiographic loosening, wear, and radiolucencies.

Patients and Methods We retrospectively followed 25 patients (27 hips) with extensive acetabular defects. No patient was lost to followup. Two patients died during followup. Minimum followup was 3 years (mean, 8.8 years; range, 3-14.1 years).

Results Three patients (three hips) underwent repeat revision surgery and another two patients (two hips) had radiographically loose hips. The 10 -year survival rate
\end{abstract}

Each author certifies that he or she has no commercial associations (eg, consultancies, stock ownership, equity interest, patent/licensing arrangements, etc) that might pose a conflict of interest in connection with the submitted article.

Each author certifies that his or her institution has approved the human protocol for this investigation, that all investigations were conducted in conformity with ethical principles of research.

N. van Egmond, D. C. J. De Kam, J. W. M. Gardeniers,

B. W. Schreurs $(\square)$

Department of Orthopaedics 357, Radboud University Nijmegen

Medical Centre, PO Box 9101, 6500 HB Nijmegen,

The Netherlands

e-mail: B.Schreurs@orthop.umcn.nl was $88 \%$ (95\% confidence interval, $74.2 \%-100 \%$ ) with the end point acetabular revision for any reason and 95\% (95\% confidence interval, $86.0 \%-100 \%$ ) with the end point acetabular revision for aseptic loosening. The mean HHSs were 55 points before surgery and 72 points postoperatively.

Conclusions Acetabular reconstruction with impaction bone grafting and a cemented cup is a reliable technique with a 10-year survival rate of $88 \%$ in patients with extensive acetabular deficiencies.

Level of Evidence Level IV, case series. See the Guidelines for Authors for a complete description of levels of evidence.

\section{Introduction}

The most challenging aspect of acetabular revision is managing the bone stock loss and creating a stable reconstruction with long-term durability. Reports show that restoring the normal biomechanical anatomy enhances survival and function [19, 42]. Several techniques are described to reconstruct extensive acetabular defects, including structural grafts $[20,21,26,36,46]$, reinforcement rings and cages [2, 28, 37, 38, 51, 52], placement of the acetabular component in a high hip center [12, 29, 45], jumbo cups [13, 24, 25, 35, 50], bilobed cups [1, 3, 8, 14, 30], use of trabecular metal acetabular augments $[4,32]$, and the triflange cup $[9,17]$. Results vary among these techniques (Table 1).

We believe that restoring acetabular bone stock loss is essential for better survival rates, clinical function and pain, and radiographic appearances in revision hip arthroplasty. Also, restoring bone stock loss provides a better starting point for any subsequent revision. For almost 


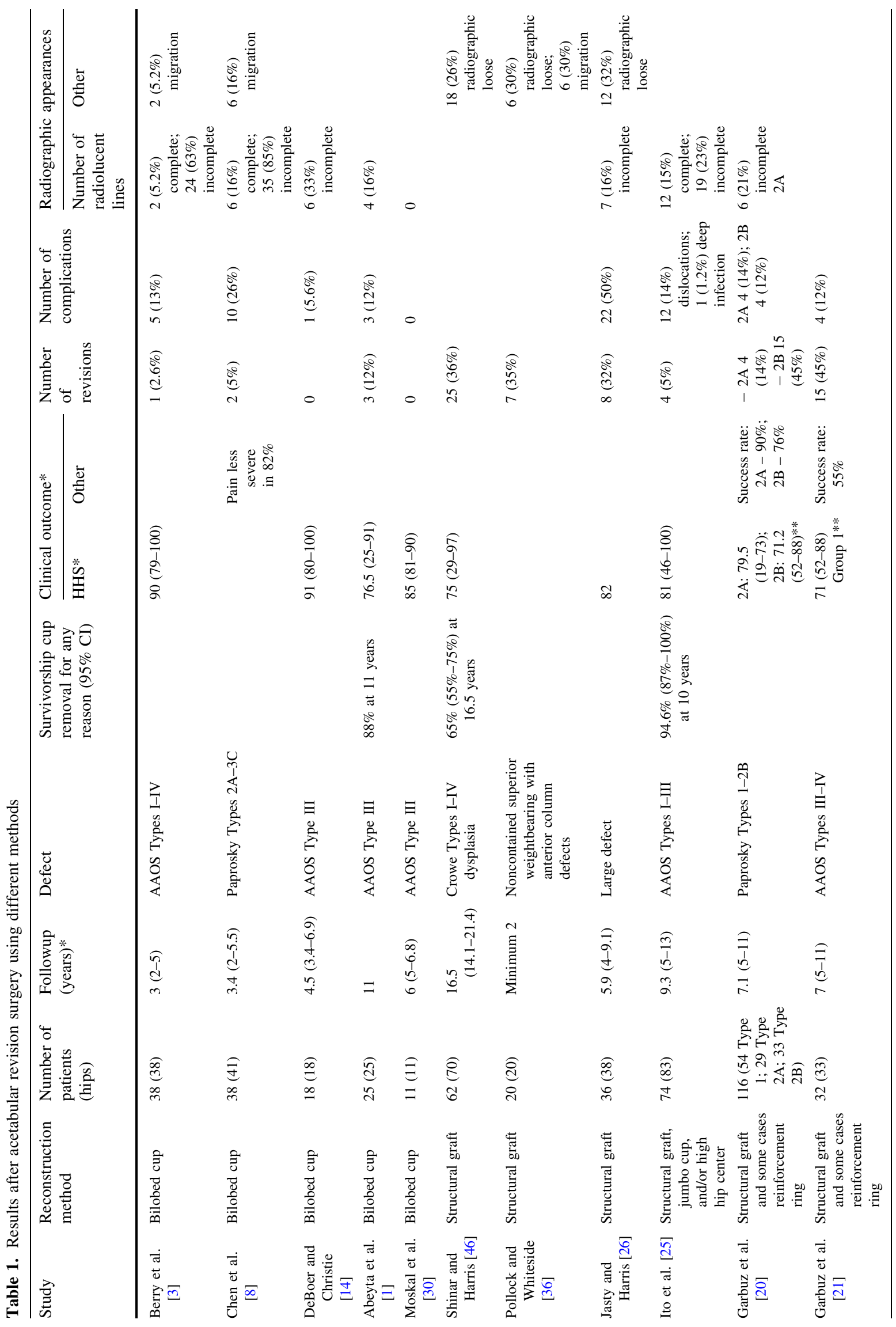




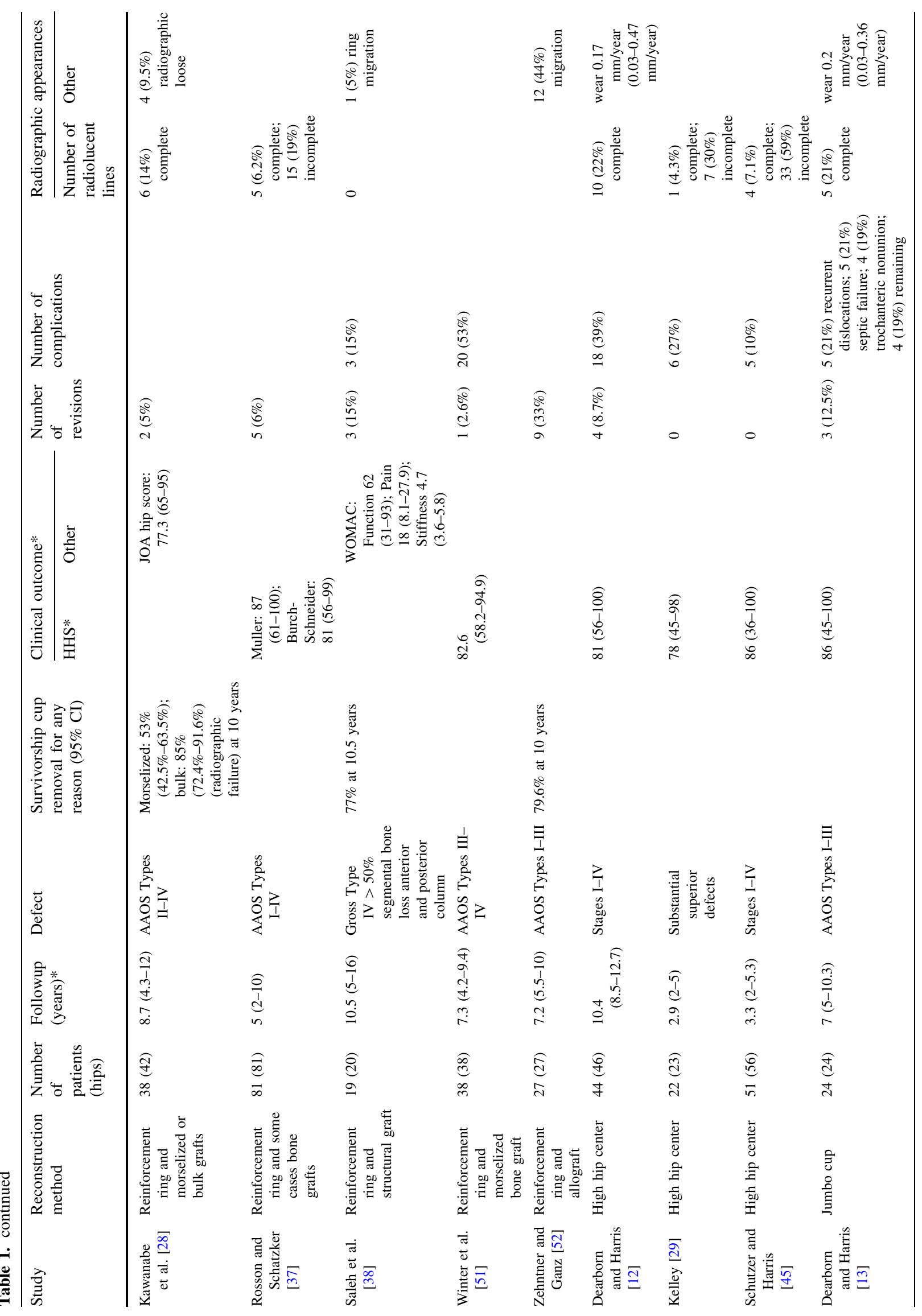




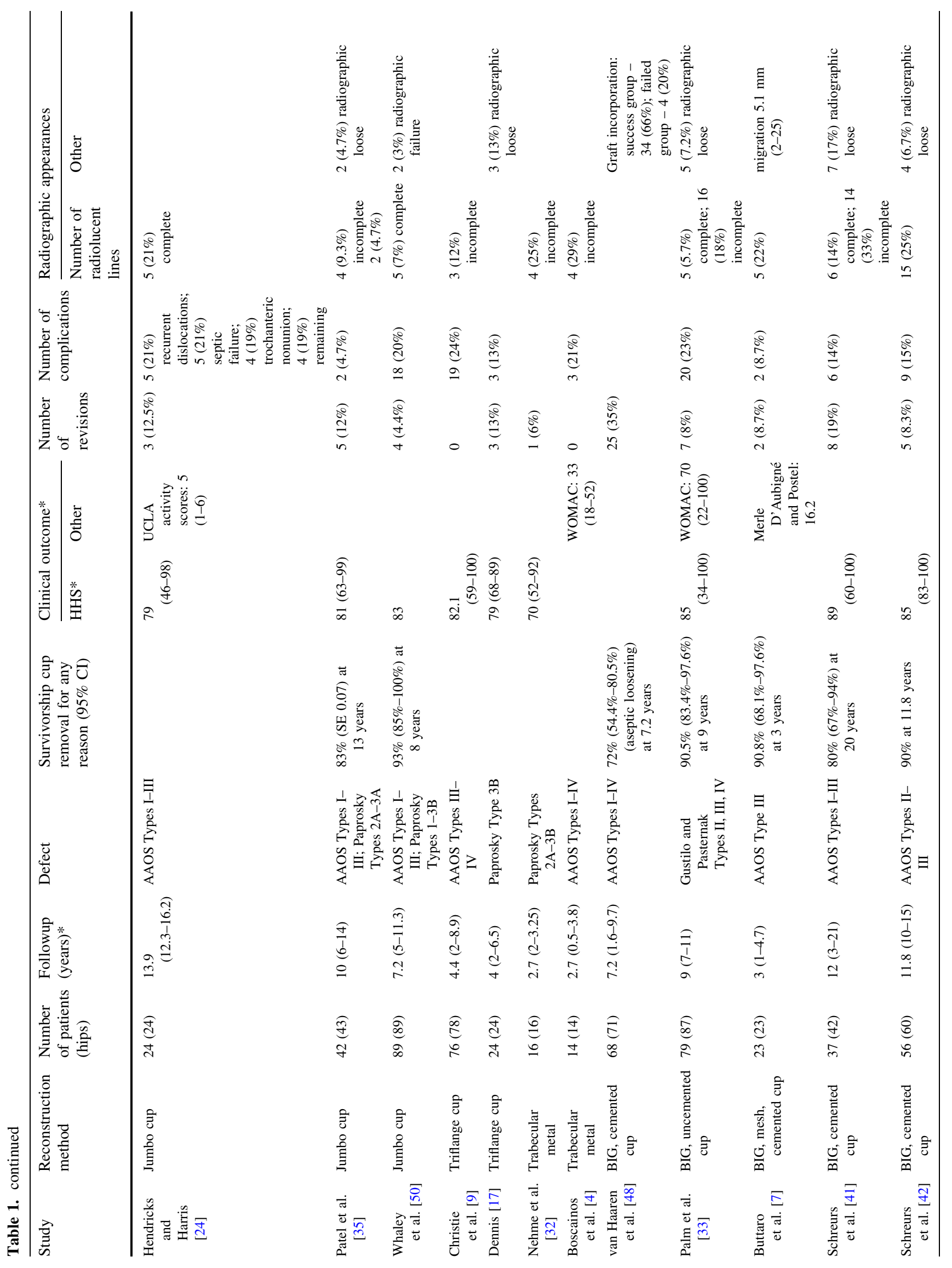




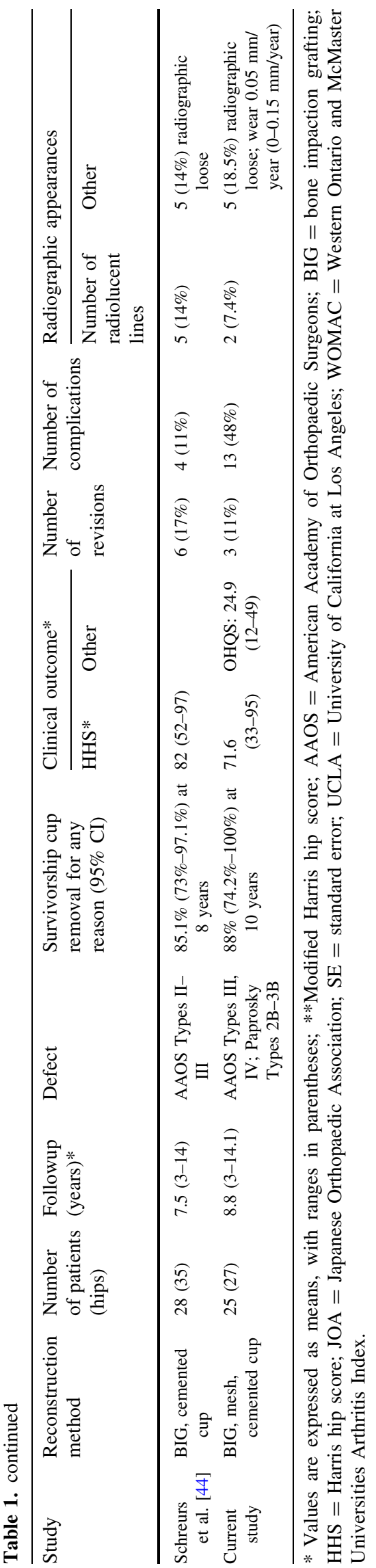

30 years, we have used bone impaction grafting in combination with a cemented cup to reconstruct the acetabulum during primary and revision procedures in patients with acetabular defects [40-44, 49]. Survival rates of $90.8 \%$ have been reported at short-term followup (24-56 months) [7], $72 \%$ to $90 \%$ at midterm $(7.2-7.5$ years) $[43,48]$, and $80 \%$ to $94 \%$ at long term (11.8-20 years) $[40,41,44,49]$. With more extensive defects, there is controversy whether bone impaction grafting can be used. One study reported a survival rate of $72 \% ; 70 \%$ of the reconstructed cups had an American Academy of Orthopaedic Surgery (AAOS) Type III or IV bone defect [48].

We studied a group of patients with acetabular reconstructions with bone impaction grafting for extensive bone deficiency. All had a reconstruction with a large-rim mesh. We determined the (1) survival rates with the end point revision for any reason, aseptic revision, and radiographic loosening; (2) VAS pain score, HHS [22], and the OHQS [11]; (3) number of repeat revisions; (4) complications; and (5) radiographic loosening, wear, and radiolucencies.

\section{Patients and Methods}

Between January 1993 and December 2003, we performed 27 acetabular revisions in 25 patients with large acetabular defects using impacted bone grafts and a cemented cup. During this period, we performed 358 acetabular revisions in total. We did not use any other technique for severe deficiency reconstruction. The indication for the revision was aseptic loosening of the acetabular component in 22 hips, septic loosening in four hips, and one with resection arthroplasty greater than 6 months. For this study, we included patients only with an X-Change large-rim mesh (Stryker-Howmedica, Newbury, UK). We had not classified the severity of the defects at the time of surgery and therefore included only patients who had 6 weeks of postoperative bed rest or 3 weeks of bed rest together with 3 weeks of nonweightbearing mobilization; these were all patients who were judged to have the most severe defects. All ages and all indications for revision THA were included. The number of previous revisions was not an exclusion criterion. Five men (five hips) and 20 women (22 hips) were included, with a mean age of 63 years (range, 42-82 years) at the time of the index revision procedure. Fifteen revisions were on the right side and 12 on the left side. The minimum followup for all patients was 3 years (mean, 8.8 years; range, 3-14.1 years). No patient was lost to followup. During followup, two patients (two hips) died from causes not related to the revision procedure at 1 year and 7.5 years postoperatively. One patient died from hepatic failure; however, she had aseptic loosening of her acetabular reconstruction but did not 
undergo reoperation because of her poor medical condition. The second patient had a well-functioning arthroplasty. The minimum followup of the surviving patients (not deceased, hips not revised) was 4 years (mean, 9.7 years; range, 4-14.1 years).

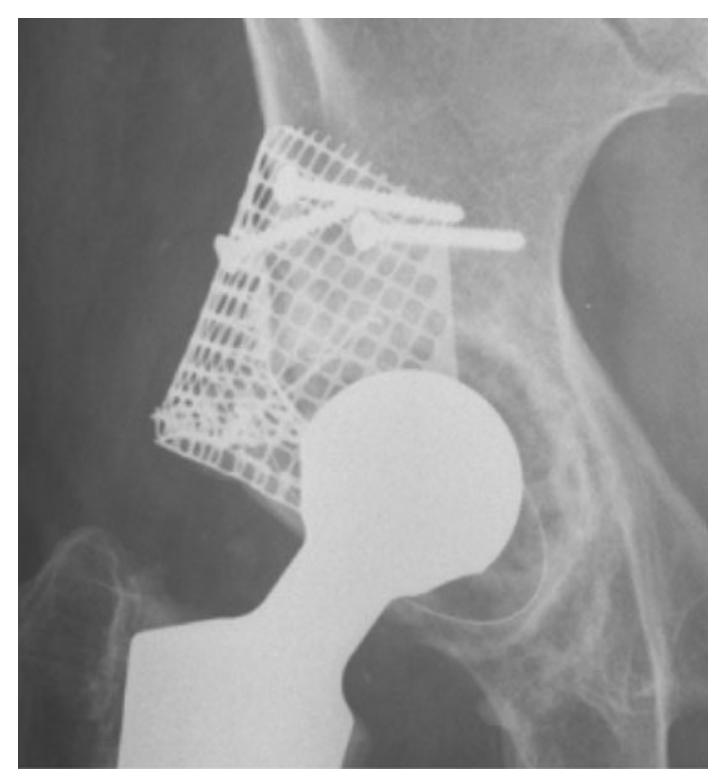

Fig. 1 An example of an X-Change large-rim mesh is shown.
We retrospectively classified all defects using the AAOS system [10]: 25 had a Type III defect and two had a Type IV defect. According to the classification of Paprosky et al. [34], four hips had Type 2B, 14 hips had Type 3A, and nine hips had Type 3B defects. One metal mesh was used in nine hips, two meshes were used in 16 hips, and three meshes were used in two hips. Three to nine screws were used for fixation of the meshes.

All revisions were performed by two surgeons (BWS, JWMG). The acetabular revision was combined with a femoral revision in 22 cases. The average surgical time was 240 minutes (range, 120-425 minutes). A posterolateral approach was used in all patients. The bone bed was rinsed with pulse lavage after removal of the cup, cement, and interface. Interface tissue was sent for cultures and frozen sections. Segmental bone defects of the acetabulum were contained with one or more metal meshes fixated with screws. In all cases, the superolateral wall defect was reconstructed with a large-rim X-Change metal mesh (Fig. 1). If needed, medial wall meshes were used (Fig. 2 A). Next, sclerotic areas were perforated by multiple $2-\mathrm{mm}$ drill holes, and impaction bone grafting was performed. Bone chips with a diameter of 0.7 to $1.0 \mathrm{~cm}$ were made by morselizing femoral head allografts from the local bone bank with a rongeur or a bone mill (Fig. 2B). The chips were impacted with metal impactors (X-Change system;

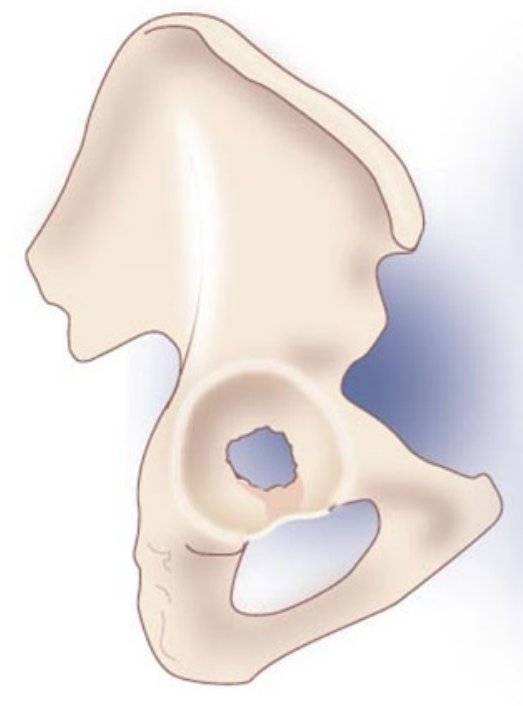

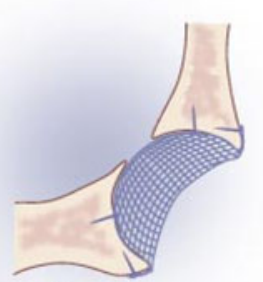

A

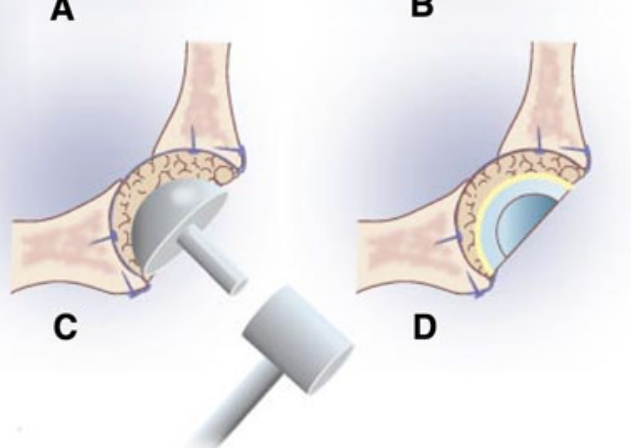

Fig. 2A-D (A) A flexible stainless steel mesh is used to close the segmental defects. (B) For acetabular reconstruction, 7- to 10-mm diameter fresh-frozen morselized bone chips are impacted using end metal impactors in several diameters. (C) With a hammer, the bone chips, layer by layer, are compressed tightly. (D) Bone cement is introduced in a relatively viscous state and is pressurized to force bone cement into the graft. Reconstruction of the cup after cup placement at the anatomic level is shown [6]. (Reproduced with permission from Busch VJ, Gardeniers JW, Slooff TJ, Veth RP, Schreurs BW. [Favourable long-term results from cemented total hip arthroplasty combined with acetabular bone impaction grafting in patients under the age of 50] [in Dutch]. Ned Tijdschr Geneeskd. 2007;151:1935-1940.). 
Stryker Howmedica) and a mallet (Fig. 2C). The original center of rotation of the hip was reconstructed with the transverse acetabular ligament as a reference mark. We used one to five femoral heads for the reconstruction. Five patients needed an additional structural graft for proper fixation of the wire meshes. The structural grafts were placed behind the meshes. All cups were implanted with antibiotic-loaded bone cement (Surgical Simplex ${ }^{\mathbb{R}}$; Stryker Howmedica) with third-generation cementing techniques and with a conventional full high molecular weight polyethylene (HMWPE) cup (Fig. 2D). Eleven Muller 32-mm cups (Sulzer, Winterthur, Switzerland), eight Exeter/Contemporary 28-mm cups (Stryker Howmedica), and eight Charnley/Elite 28-mm cups (DePuy, Leeds, UK) were used. The choice of the definitive cup size was made by the last trial cup and reamer used. The inner diameter was standard $28 \mathrm{~mm}$, but occasionally a 32-mm cup was used to create a more stable situation or when there was a high risk of dislocation. We used only the planned operation technique; no alteration of surgical plans was necessary $[6$, 42] (Fig. 3).

All patients received antibiotics (cefazolin) after results of intraoperative cultures were obtained. On indication and guided by these cultures, prolonged antibiotics were prescribed. NSAIDs were given for 7 days to prevent heterotopic ossification. All patients received thrombosis prophylaxis with low-molecular-weight heparin for 6 weeks or, before 1999, with oral anticoagulation for 3 months. Twenty patients (21 hips) had bed rest for 6 weeks. In five patients (six hips), an initial 6-week period
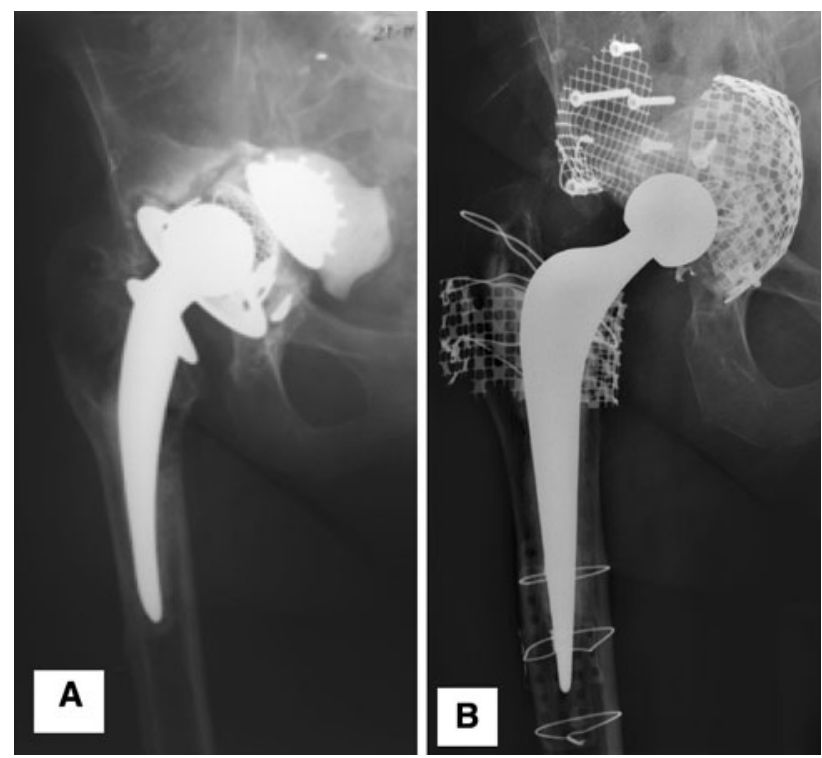

Fig. 3A-B (A) An AP radiograph shows the right acetabulum of a patient who had aseptic loosening of two cups in the pelvis with an AAOS Type III and Paprosky Type 3B defect. (B) Twelve years after revision, no radiolucent lines, no migration, and incorporation of the graft can be seen of bed rest was planned, but nonweightbearing mobilization was used for the last 3 weeks. Under the supervision of a physical therapist, mobilization with partial (10\%) weightbearing was begun at 6 weeks and 50\% weightbearing with crutches at 12 weeks. Full weightbearing was allowed after 18 weeks after surgery.

All patients were followed on a regular basis or until death; data of the two patients who died were included. Routine followups were scheduled at 6 weeks; 3,6 , and 12 months; and yearly or biannually thereafter. At our outpatient clinic, independent student researchers not participating in the treatment performed clinical analyses using the HHS [22], the OHQS (since 1998) [11], and a VAS for pain. The radiographic examination consisted of AP and lateral radiographs of the hip or pelvis. Preoperative and postoperative radiographs were available for all patients. At last review, three patients (three hips) were unable to return for the clinical review owing to their advanced age and general health limitations. By telephone interview, none had complaints of the reconstructed hip and none have had additional surgery on the hip. The last radiographs for these three patients $(4.0,4.4$, and 7.8 years postoperatively) were included in the radiographic review. The preoperative HHSs were available for 15 patients and postoperative HHSs were available for 20 patients [22]. The preoperative OHQSs were available for two patients and postoperative scores were available for 19 [11]. Postoperative VAS scores for pain $(0=$ best score, $100=$ worst score) were available for 18 patients.

Three investigators (NvE, DCJK, BWS) independently evaluated the radiographs for signs of incorporation, radiolucent lines, cup position, heterotopic ossification, and polyethylene wear. Whenever we differed regarding evaluation of a radiograph, we reviewed it again together without knowing our previous scores for that radiograph. We then reached agreement on the score, which, in all instances, was one of the scores from the previous evaluation; we did not determine interobserver variability of any measures. Radiographic evidence of incorporation was defined as equal radiodensity of the graft and host bone with a continuous trabecular pattern throughout, as described by Slooff et al. [47]. Radiolucent lines greater than $2 \mathrm{~mm}$ in width were identified in the three zones of DeLee and Charnley [16]. Radiolucent lines were defined as stable or progressive in width and extent. Position of the cup was defined as neutral in $45^{\circ}\left( \pm 10^{\circ}\right)$, vertical in $56^{\circ}$ or greater, and horizontal in $34^{\circ}$ or less, according to Salvati et al. [39]. Heterotopic ossification was classified according to Brooker et al. [5]. Polyethylene wear was calculated using the Dorr method as described by Ebramzadeh et al. [18].

Failure was defined as the need for repeat revision of the acetabular component for any reason, and radiographic failure was defined as radiolucent lines in all three zones or 
tilting of the cup $8^{\circ}$ or greater and migration of $5 \mathrm{~mm}$ or greater relative to the interteardrop line, in any direction on the AP radiographs of the pelvis.

Survivorship analysis was performed using the KaplanMeier method [27] with the end points of revision for any reason, aseptic revision, and radiographic loosening. SPSS v 16.0 (SPSS Benelux BV, IBM Company Nieuwegein, The Netherlands) was used for statistical analysis.

\section{Results}

The probability of survival of the acetabular component at 10 years, with removal of the cup for any reason as the end point is $87.6 \%$ (95\% confidence interval [CI], 74.2\%$100 \%$ ) (Fig. 4). With revision for aseptic loosening as the end point, the survival rate is $95.2 \%$ (95\% CI, 86.0\%$100 \%$ ) at 10 years (Fig. 5). The survival rate with

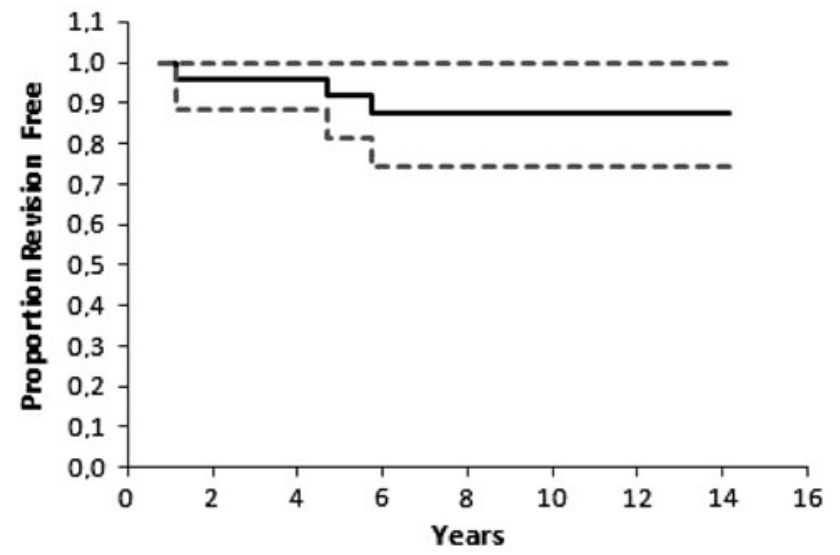

Fig. 4 A Kaplan-Meier curve shows survival with an end point of revision of the cup for any reason. Dotted lines $=95 \%$ CI. At 10 years, the survival rate was $87.6 \%$ (95\% CI, $74.2 \%-100 \%)$.

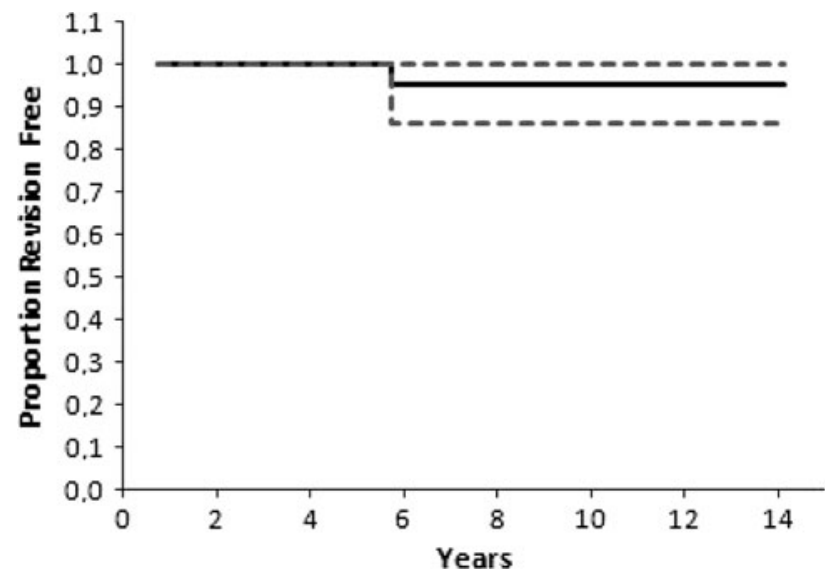

Fig. 5 A Kaplan-Meier curve shows survival with an end point of revision of the cup for aseptic loosening. Dotted lines $=95 \%$ CI. The survival rate was $95.2 \%(95 \% \mathrm{CI}, 86.0 \%-100 \%)$ at 10 years. radiographic loosening as the end point is $77.2 \%$ (95\% CI, $59.0 \%-95.4 \%$ ) at 10 years (Fig. 6).

The surviving patients had improved clinical scores after acetabular revision with bone impaction grafting and a cemented cup, even after a mean followup of 9.7 years (Table 2).

Three hips (11\%) had failed results and underwent rerevision or removal of the implant. The use of a structural graft did not influence the survival rate. One hip failed 1.1 years after surgery. This patient had two previous revision surgeries. There was a massive posterosuperior defect (AAOS Type III and Paprosky Type 3B). Against our protocol and advice, the patient started full weightbearing 4 weeks postoperatively. The graft did not incorporate and the cup subluxated. At 1.1 years, conversion to a resection arthroplasty was done. One hip failed 4.7 years after surgery owing to culture-proven septic loosening. The index revision was the fourth revision.

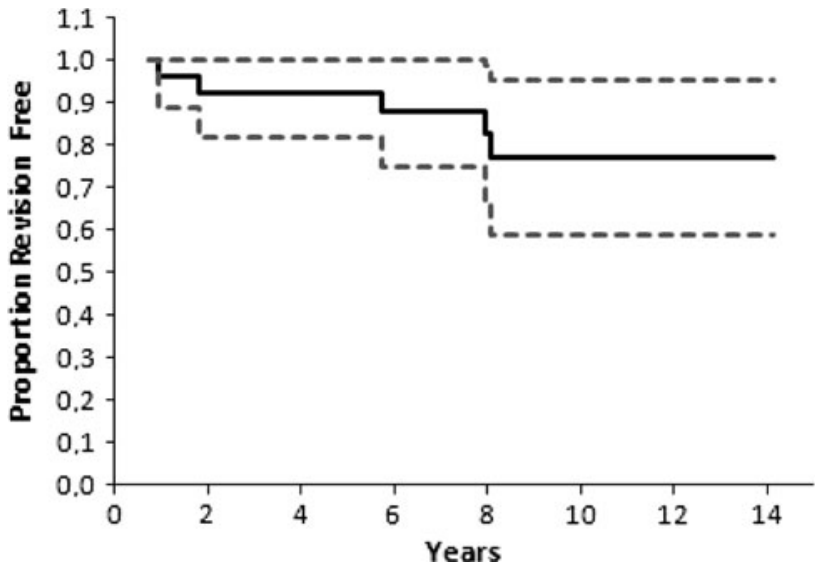

Fig. 6 A Kaplan-Meier curve shows survival with an end point of radiographic loosening of the cup. Dotted lines $=95 \%$ CI. The survival rate was $77.2 \%$ (95\% CI, 59.0\%-95.4\%) at 10 years.

Table 2. Clinical outcomes

\begin{tabular}{lllll}
\hline Score & $\begin{array}{l}\text { Number of } \\
\text { patients (hips) } \\
\text { available }\end{array}$ & Median & Mean & Range \\
\hline Preoperative HHS & $15(15)$ & 51 & 55.3 & $30-95$ \\
Postoperative HHS* & $18(20)$ & 73.5 & 71.6 & $33-95$ \\
Preoperative OHQS & $2(2)$ & 26 & 26 & $17-35$ \\
Postoperative OHQS* & $17(19)$ & 23 & 24.9 & $12-49$ \\
Postoperative VAS pain & $17(18)$ & 0 & 11.7 & $0-80$ \\
$\quad$ score at rest* & & 0 & 14.7 & $0-70$ \\
Postoperative VAS pain & $17(18)$ & & & \\
score on movement* & & & & \\
\hline Surve
\end{tabular}

* Surviving hips in living patients who did not have a repeat revision; HHS = Harris hip score; OHQS = Oxford Hip Questionnaire Score; $\mathrm{VAS}=$ visual analog scale. 
Table 3. Complications

\begin{tabular}{|c|c|c|c|}
\hline Complications & Number & Treatment & Outcome \\
\hline \multicolumn{4}{|l|}{ Cup failures } \\
\hline Unstable cup & 1 & Resection arthroplasty & Resection arthroplasty \\
\hline Septic loosening & 1 & Resection arthroplasty & Resection arthroplasty \\
\hline Aseptic loosening & 1 & Cup rerevision & No additional problems \\
\hline \multicolumn{4}{|l|}{ Revision femoral component } \\
\hline Recurrent dislocation & 1 & Femoral stem rerevision & No additional problems \\
\hline $\begin{array}{l}\text { Perioperative unnoticed distal stem } \\
\text { perforation of the femoral shaft }\end{array}$ & 1 & Femoral stem rerevision & No additional problems \\
\hline \multicolumn{4}{|l|}{ Perioperative complication } \\
\hline Periprosthetic fracture & 3 & Open reduction and internal fixation & Stable reconstruction \\
\hline \multicolumn{4}{|l|}{ Postoperative complication } \\
\hline Neurapraxia of peroneal nerve & 1 & Observation & Permanent partial motor paralysis \\
\hline Dislocation hip & 2 & Newport brace & No additional problems \\
\hline Delayed wound healing with effusion & 1 & Observation & No additional problems \\
\hline Early septic loosening & 1 & $\begin{array}{l}\text { Observation (owing to patient's poor } \\
\text { medical condition) }\end{array}$ & \\
\hline
\end{tabular}

The patient had one previous revision because of septic loosening and three revisions because of aseptic loosening. He was treated with a resection arthroplasty. The third acetabular component was revised owing to aseptic loosening after the patient fell on the floor 5.8 years postoperatively and a cup revision was performed.

There were no major intraoperative complications during acetabular reconstruction. There were 13 complications in total (Table 3).

Five hips had radiographic signs of loosening based on migration; three of these were described above and had repeat revision surgeries. The revised hips were considered radiographically loose after $1.0,1.9$, and 5.8 years. Two hips in two patients, radiographically loose after 8.0 and 8.1 years postoperatively, were not revised. These two patients had complaints, but no repeat revision surgery was planned because of their old age and poor medical conditions. We observed no failures attributable to progressive radiolucent lines. All grafts (including structural grafts) incorporated. Fourteen hips had polyethylene wear however, the wear was excessive $(>0.1 \mathrm{~mm} / \mathrm{year})$ in only three. The mean wear was $0.05 \mathrm{~mm} / \mathrm{year}$ (range, $0-0.15 \mathrm{~mm} /$ year). Heterotopic ossifications were seen in nine hips. Five hips had Class I ossifications, three had Class II, and one had Class III ossifications.

\section{Discussion}

Managing severe acetabular bone loss in revision arthroplasty can be challenging. Various techniques have been described to manage these deficiencies, with varying results (Table 1). Bone impaction grafting is one of the few reconstruction techniques that restores the loss of bone stock and allows creation of an anatomic and biomechanical natural center of rotation. It has reported survival rates between $72 \%$ and $94 \%$ [40, 41, 43, 44, 48, 49]. In the more extensive defects there is controversy whether bone impaction grafting can be used. One study found a survival rate of $72 \%$ [48]. Of the 20 acetabular components revised in patients in that study [48], 70\% had an AAOS Type III or IV bone defect. In our current study, in a group of patients with acetabular revision with bone impaction grafting for extensive bone deficiency, we determined the (1) survivor rates, (2) pain relief and function, (3) number of rerevisions, (4) complications, and (5) radiographic appearances.

Our study has some limitations. First, we had a limited number of patients. However, no patients were lost to followup and the worst-case scenario survival rate [31] was $88 \%$ at 8.8 years. Also, we believe the group is representative because all ages and all revision and primary indications for the revision and primary arthroplasties are included. Second, we retrospectively classified the severity of the defects and therefore the indications for surgery were not strictly based on a given level of severity. However, for this study, we included only patients who had 6 weeks of bed rest and the use of the X-Change large-rim mesh, both of which we used only for patients needing more extensive reconstructions. Bed rest was prescribed only for patients with severe defects in whom we believed, at the time, early mobilization would jeopardize the stability of the reconstruction. Obviously, a 6-week period of bed rest is a disadvantage of this technique. Third, with a mean 
followup of 8.8 years our followup is midterm. For final proof long-term data are needed. Fourth, we unfortunately had some missing clinical data, but available data show a trend that the clinical function and pain are improving after acetabular reconstruction with bone impaction grafting. We have used the OHQS [11] only the past few years and therefore do not have previous data.

The 10-year survival rates of the reconstructions in this study were $88 \%$ with removal of the cup for any reason as the end point, $95 \%$ with revision for aseptic loosening as the end point, and $77 \%$ with radiographic loosening as the end point at a mean followup of 8.8 years (range, 3-14.1 years). Survival rates vary among the different techniques used in large acetabular defects (Table 1). Abeyta et al. [1], who investigated the bilobed cup in large reconstructions, reported a survival rate of $88 \%$ for 25 patients with an AAOS Type III defect after a followup of 11 years. The study was limited by a high number of lost and deceased patients. In the worst-case scenario, the overall survival rate would decrease to $48 \%$. Reconstruction with a structural graft has good short-term survival rates (zero revisions at a mean of 17.3 months) [23], but middle and long-term (2-16.5 years) failure rates of $32 \%$ to $45 \%$ are reported for these grafts [21, 26, 36, 46]. Trabecular metal acetabular augments are now commonly used in the United States for reconstruction of large acetabular defects. Several studies show low failure rates of $0 \%$ to $6 \%[4,32]$, but the followup is short (mean, 2.7 years) and the number of patients is small [4, 32]. Among 71 revisions with AAOS Types I to IV defects using bone impaction grafting and a cemented cup, van Haaren et al. found 25 hips (35\%) needed repeat revision [48]. The survival rate after 7.2 years with repeat revision with aseptic loosening as the end point was $72 \%(95 \% \mathrm{CI}$, $54.4 \%-80.5 \%$ ). However, greater than $50 \%$ of their aseptic revisions were based on cup loosening out of the cement mantle, which is an unusual failure mechanism.

In line with the literature, patients had better postoperative clinical scores after acetabular reconstruction with bone impaction grafting and a cemented cup (Table 1) [33, 40, 41, 43, 44, 49].

Three cups (11\%) needed repeat revision. Jasty and Harris [26] reported eight $(32 \%)$ of their patients needed repeat revision by a mean followup of 5.9 years using structural femoral head allografts to reconstruct large acetabular bone defects. The repeat revision rate of Abeyta et al. [1] was $12 \%$, similar to ours, using a bilobed cup in the reconstruction of acetabular deficiencies.

There were 13 (48\%) complications. Different complication rates are described using bone impaction grafting techniques (Table 1). Buttaro et al. [7] described only two $(8.7 \%)$ reoperations and two $(8.7 \%)$ other complications (infection and dislocation). Palm et al. [33] reported
$20(23 \%)$ complications. However, this report was based on bone impaction grafting with noncemented cups. Also, high complications rates are reported using structural grafts $(50 \%)$ [26], jumbo cups $(21 \%)$ [13, 24, 50], and the triflange cup (24\%) [9].

In addition to the three hips needing repeat revision, another two cups were considered radiographically loose. In the other cases, no radiolucent lines were seen, which is remarkable. This could be attributed to the unique cup, graft, cement, and bone interface. De Kam et al. [15] reported fewer radiolucent lines were seen in patients with impacted bone grafts in primary THA. In a study of revision arthroplasty using impaction grafting, Buttaro et al. [7] also reported no additional radiolucent lucencies except in patients with hips needing repeat revision.

We believe the proper function and the survival of an acetabular reconstruction in revision surgery depend on achieving adequate fixation of the new component, restoring the anatomic center of rotation of the hip, and restoring bone stock loss. We believe that for extensive reconstructions with bone impaction grafting, an aftertreatment protocol of 6 weeks of bed rest, or 3 weeks of bed rest and 3 weeks nonweightbearing mobilization, is crucial for acceptable survival rates and improving clinical function and pain. In the study by Buttaro et al. [7] regarding the outcome of larger defects and bone impaction grafting, 6 weeks of unloading with crutches was used and at 3 years, the survival rate was $90.8 \%$. However, one of their failed results involved a patient who decided to fully load the reconstruction and this resulted in failure, as occurred with one patient with failed results in our study.

Acetabular reconstruction using impaction bone grafting together with a cemented cup is a reliable technique with favorable midterm survival rates and clinical function in patients with massive acetabular defects. Impacted bone grafting is an acetabular revision technique that restores bone stock loss and allows reconstruction of the normal anatomic biomechanics of the hip.

Open Access This article is distributed under the terms of the Creative Commons Attribution Noncommercial License which permits any noncommercial use, distribution, and reproduction in any medium, provided the original author(s) and source are credited.

\section{References}

1. Abeyta PN, Namba RS, Janku GV, Murray WR, Kim HT. Reconstruction of major segmental acetabular defects with an oblong-shaped cementless prosthesis: a long-term outcomes study. J Arthroplasty. 2008;23:247-253.

2. Berry DJ, Müller ME. Revision arthroplasty using an antiprotrusio cage for massive acetabular bone deficiency. $J$ Bone Joint Surg Br. 1992;74:711-715.

3. Berry DJ, Sutherland CJ, Trousdale RT, Colwell CW Jr, Chandler HP, Ayres D, Yashar AA. Bilobed oblong porous coated 
acetabular components in revision total hip arthroplasty. Clin Orthop Relat Res. 2000;371:154-160.

4. Boscainos PJ, Kellett CF, Maury AC, Backstein D, Gross AE. Management of periacetabular bone loss in revision hip arthroplasty. Clin Orthop Relat Res. 2007;465:159-165.

5. Brooker AF, Bowerman JW, Robinson RA, Riley LH Jr. Ectopic classification following total hip replacement: incidence and a method of classification. J Bone Joint Surg Am. 1973;55:16291632.

6. Busch VJ, Gardeniers JW, Slooff TJ, Veth RP, Schreurs BW. [Favourable long-term results from cemented total hip arthroplasty combined with acetabular bone impaction grafting in patients under the age of 50] [in Dutch]. Ned Tijdschr Geneeskd. 2007;151:1935-1940.

7. Buttaro MA, Comba F, Pusso R, Piccaluga F. Acetabular revision with metal mesh, impaction bone grafting, and a cemented cup. Clin Orthop Relat Res. 2008;466:2482-2490.

8. Chen WM, Engh CA Jr, Hopper RH Jr McAuley JP, Engh CA. Acetabular revision with use of a bilobed component inserted without cement in patients who have acetabular bone-stock deficiency. J Bone Joint Surg Am. 2000;82:197-206.

9. Christie MJ, Barrington SA, Brinson MF, Ruhling ME, DeBoer DK. Bridging massive acetabular defects with the triflange cup: 2- to 9-year results. Clin Orthop Relat Res. 2001; 393:216-227.

10. D'Antonio JA, Capello WN, Borden LS, Bargar WL, Bierbaum BF, Boettcher WG, Steinberg ME, Stulberg SD, Wedge JH. Classification and management of acetabular abnormalities in total hip arthroplasty. Clin Orthop Relat Res. 1989;243:126-137.

11. Dawson J, Fitzpatrick R, Carr A, Murray D. Questionnaire on the perceptions of patients about total hip replacement. J Bone Joint Surg Br. 1996;78:185-190.

12. Dearborn JT, Harris WH. High placement of an acetabular component inserted without cement in a revision total hip arthroplasty: results after a mean of ten years. J Bone Joint Surg Am. 1999;81:469-480.

13. Dearborn JT, Harris WH. Acetabular revision arthroplasty using so-called jumbo cementless components: an average 7-year follow-up study. J Arthroplasty. 2000;15:8-15.

14. DeBoer DK, Christie MJ. Reconstruction of the deficient acetabulum with an oblong prosthesis: three- to seven-year results. J Arthroplasty. 1998;13:674-680.

15. de Kam DC, Gardeniers JW, Hendriks JC, Veth RP, Schreurs BW. Cemented polyethylene cups in patients younger than 40 years. Clin Orthop Relat Res. 2009;467:1753-1764.

16. DeLee JG, Charnley J. Radiological demarcation of cemented sockets in total hip replacement. Clin Orthop Relat Res. 1976; 121:20-32.

17. Dennis DA. Management of massive acetabular defects in revision total hip arthroplasty. J Arthroplasty. 2003;18(3 suppl 1): $121-125$.

18. Ebramzadeh E, Sangiorgio SN, Lattuada F, Kang JS, Chiesa R, McKellop HA, Dorr LD. Accuracy of measurement of polyethylene wear with use of radiographs of total hip replacement. J Bone Joint Surg Am. 2003;85:2378-2384.

19. Etienne G, Bezwada HP, Hungerford DS, Mont MA. The incorporation of morselized bone grafts in cementless acetabular revisions. Clin Orthop Relat Res. 2004;428:241-246.

20. Garbuz D, Morsi E, Gross AE. Revision of the acetabular component of a total hip arthroplasty with a massive structural allograft: study with a minimum five-year follow-up. J Bone Joint Surg Am. 1996;78:693-697.

21. Garbuz D, Morsi E, Mohamed N, Gross AE. Classification and reconstruction in revision acetabular arthroplasty with bone stock deficiency. Clin Orthop Relat Res. 1996;324:98-107.
22. Harris WH. Traumatic arthritis of the hip after dislocation and acetabular fractures: treatment by mold arthroplasty. An endresult study using a new method of result evaluation. J Bone Joint Surg Am. 1969;51:737-755.

23. Harris WH. Allografting in total hip arthroplasty in adults with severe acetabular deficiency including a surgical technique for bolting the graft to the ilium. Clin Orthop Relat Res. 1982;162: $150-164$.

24. Hendricks KJ, Harris WH. Revision of failed acetabular components with use of so-called jumbo noncemented components: a concise follow-up of a previous report. J Bone Joint Surg Am. 2006;88:559-563.

25. Ito H, Matsuno T, Aoki Y, Minami A. Acetabular components without bulk bone graft in revision surgery: a 5- to 13-year follow-up study. J Arthroplasty. 2003;18:134-139.

26. Jasty M, Harris WH. Salvage total hip reconstruction in patients with major acetabular bone deficiency using structural femoral head allografts. J Bone Joint Surg Br. 1990;72:63-67.

27. Kaplan EL, Meier P. Nonparametric estimation from incomplete observations. J Am Stat Assoc. 1958;53:457-481.

28. Kawanabe K, Akiyama H, Onishi E, Nakamura T. Revision total hip replacement using the Kerboull acetabular reinforcement device with morsellised or bulk graft: results at a mean follow-up of 8.7 years. J Bone Joint Surg Br. 2007;89:26-31.

29. Kelley SS. High hip center in revision arthroplasty. J Arthroplasty. 1994;9:503-510.

30. Moskal JT, Higgins ME, Shen J. Type III acetabular defect revision with bilobed components: five-year results. Clin Orthop Relat Res. 2008;466:691-695.

31. Murray DW, Britton AR, Bulstrode CJ. Loss to follow-up matters. J Bone Joint Surg Br. 1997;79:254-257.

32. Nehme A, Lewallen DG, Hanssen AD. Modular porous metal augments for treatment of severe acetabular bone loss during revision hip arthroplasty. Clin Orthop Relat Res. 2004;429: 201-208.

33. Palm L, Jacobsson SA, Kvist J, Lindholm A, Öjersjö A, Ivarsson I. Acetabular revision with extensive allograft impaction and uncemented hydroxyapatite-coated implants: results after 9 (7-11) years follow-up. J Arthroplasty. 2007;22:1083-1091.

34. Paprosky WG, Perona PG, Lawrence JM. Acetabular defect classification and surgical reconstruction in revision arthroplasty: a 6-year follow-up evaluation. J Arthroplasty. 1994;9:33-44.

35. Patel JV, Masonis JL, Bourne RB, Rorabeck CH. The fate of cementless jumbo cups in revision hip arthroplasty. $J$ Arthroplasty. 2003;18:129-133.

36. Pollock FH, Whiteside LA. The fate of massive allografts in total hip acetabular revision surgery. J Arthroplasty. 1992;7:271276.

37. Rosson J, Schatzker J. The use of reinforcement rings to reconstruct deficient acetabula. J Bone Joint Surg Br. 1992;74: 716-720.

38. Saleh KJ, Jaroszynski G, Woodgate I, Saleh L, Gross AE. Revision total hip arthroplasty with the use of structural acetabular allograft and reconstruction ring: a case series with a 10 -year average follow-up. J Arthroplasty. 2000;15:951-958.

39. Salvati EA, Im VC, Aglietti P, Wilson PD Jr. Radiology of total hip replacements. Clin Orthop Relat Res. 1976;121:74-82.

40. Schreurs BW, Busch VJ, Welten ML, Verdonschot N, Slooff TJ, Gardeniers JW. Acetabular reconstruction with impaction bonegrafting and a cemented cup in patients younger than fifty years old. J Bone Joint Surg Am. 2004;86:2385-2392.

41. Schreurs BW, Slooff TJ, Buma P, Gardeniers JW, Huiskes R. Acetabular reconstruction with impacted morsellised cancellous bone graft and cement: a 10- to 15-year follow-up of 60 revision arthroplasties. J bone Joint Surg Br. 1998;80:391-395. 
42. Schreurs BW, Slooff TJ, Gardeniers JW, Buma P. Acetabular reconstruction with bone impaction grafting and a cemented cup: 20 years' experience. Clin Orthop Relat Res. 2001;393:202-215.

43. Schreurs BW, Thien TM, de Waal Malefijt MC, Buma P, Veth RP, Slooff TJ. Acetabular revision with impacted morselized cancellous bone graft and a cemented cup in patients with rheumatoid arthritis: three to fourteen-year follow-up. $J$ Bone Joint Surg Am. 2003;85:647-652.

44. Schreurs BW, van Tienen TG, Buma P, Verdonschot N, Gardeniers JW, Slooff TJ. Favorable results of acetabular reconstruction with impacted morselized bone grafts in patients younger than 50 years: a 10- to 18-year follow-up study of 34 cemented total hip arthroplasties. Acta Orthop Scand. 2001; 72:120-126.

45. Schutzer SF, Harris WH. High placement of porous-coated acetabular components in complex total hip arthroplasty. J Arthroplasty. 1994;9:359-367.

46. Shinar AA, Harris WH. Bulk structural autogenous grafts and allografts for reconstruction of the acetabulum in total hip arthroplasty: sixteen-year-average follow-up. J Bone Joint Surg Am. 1997;79:159-168.
47. Slooff TJ, Buma P, Schreurs BW, Schimmel JW, Huiskes R, Gardeniers J. Acetabular and femoral reconstruction with impacted graft and cement. Clin Orthop Relat Res. 1996;324; $108-115$.

48. van Haaren AH, Heyligers IC, Alexander FG, Wuisman PI. High rate of failure of impaction grafting in large acetabular defects. J Bone Joint Surg Br. 2007;89:296-300.

49. Welten ML, Schreurs BW, Buma P, Verdonschot N, Slooff TJ. Acetabular reconstruction with impacted morcellized cancellous bone autograft and cemented primary total hip arthroplasty: a 10- to 17-year follow-up study. J Arthroplasty. 2000;15:819-824.

50. Whaley AL, Berry DJ, Harmsen WS. Extra-large uncemented hemispherical acetabular components for revision total hip arthroplasty. J Bone Joint Surg Am. 2001;83:1352-1357.

51. Winter E, Piert R, Volkmann R, Maurer F, Eingartner C, Weise $\mathrm{K}$, Weller S. Allogeneic cancellous bone graft and BurchSchneider ring for acetabular reconstruction in revision hip arthroplasty. J Bone Joint Surg Am. 2001;83:862-867.

52. Zehntner MK, Ganz R. Midterm results (5.5-10 years) of acetabular allograft reconstruction with the acetabular reinforcement ring during total hip revision. J Arthroplasty. 1994;9:469-479. 